\title{
Estimation of Separation Shock of the Marman Clamp System by Using a Simple Band-Mass Model
}

\author{
By Shinsuke TAKEUCHI and Junjiro ONODA \\ The Institute of Space and Astronautical Science, Sagamihara, Japan
}

(Received September 6th, 2001)

\begin{abstract}
To prevent satellite failure during inter stage separation, the magnitude of separation shock must be estimated. The Marman Clamp System is a separation joint often used to mount satellites on a launch vehicle. This paper proposes a method of estimating separation shock for the Marman Clamp System. First, experimental results are discussed and the dominant parameters of separation shock are identified for the system. Next, a simple band-mass model is proposed to estimate the system's separation shock. The differences between the model and experimental results are discussed. Radial and axial, but not tangential, separation shocks were estimated well by this method.
\end{abstract}

Key Words: Separation Shock, Marman Clamp System, Pyrotechnic Shock

\section{Nomenclature}

$D$ : bending stiffness of the cylindrical shell (See Eq. (14))

$E$ : Young's modulus of the material of the cylindrical shell

$F_{0}$ : initial force vector applied on the Marman Clamp System because of Marman band tension

$K: \quad$ stiffness matrix of the Marman Clamp System

$M$ : mass matrix of the Marman Clamp System

$M_{\mathrm{b}}$ : mass matrix of the Marman band

$Q:$ resonance magnification of the 1 DOF vibration system

$R(f)$ : ratio of shock response spectrums at frequency $f$ (See Eq. (3))

$S(f), S_{1}(f) \sim S_{4}(f)$ : values of the shock response spectrum at frequency $f$

$T:$ tension of the Marman band

$T_{0}:$ tension of the Marman band at $\theta=0$

$a:$ radius of the cylinder

$c:$ viscous coefficient of the $1 \mathrm{DOF}$ vibration system

$f:$ natural frequency of the system

$f_{\mathrm{p}}$ : frequency of maximum shock response spectrum peak

$h:$ thickness of the cylindrical shell

$k: \quad$ spring constant of the 1 DOF vibration system

$k_{\mathrm{c}}$ : spring constant relative to the radial symmetric deformation of the outside flange and inside ring per unit length along the circumference

$l$ : length of the cylindrical shell along the $x$ coordinate

$m$ : mass of the 1 DOF vibration system

$p:$ radial direction force of the Marman band

$t:$ time $w$ : displacement in the $z$ direction of the middle surface of the cylindrical shell

$x:$ axial coordinate of the cylindrical coordinates

$\boldsymbol{x}$ : nodal displacement vector

$x_{\mathrm{m}}$ : displacement of the mass of the 1 DOF vibration system

$y_{\mathrm{b}}$ : displacement of the base of the 1 DOF vibration system

$z:$ radial coordinate of the cylindrical coordinates

$\theta:$ angular coordinate of the cylindrical coordinates

$\lambda:$ minimum positive solution of Eq. (20)

$\rho:$ density of the material of the cylindrical shell

$\rho_{\mathrm{b}}$ : mass of the Marman band per unit length along the circumference

$\rho_{\mathrm{c}}$ : mass of the outside flange and inside ring per unit length along the circumference

$\mu:$ friction coefficient

$\mu_{\mathrm{s}}:$ mass of the cylindrical shell per unit area

$v$ : poisson's ratio of the material of the cylindrical shell

$\tau$ : time when the Marman band detaches

\section{Introduction}

The Marman Clamp System (MCS) is a separation joint, which is used to join and separate stages of a launch vehicle or to join a satellite to a launch vehicle. A description of MCS can be found in Ref. 1 and in the next section 2.1.

During interstage separation, a shock called "separation shock" occurs when the joint separates. To prevent a failure of the satellite as a result of separation shock, the satellite and associated instruments are designed to withstand separation shock. It is therefore vital to estimate the magnitude of separation shock during satellite design.

This paper presents methods of estimating the separation 
shock for MCS, because MCS is commonly used to join satellites to a launch vehicle. Kacena et al. and NASA have proposed methods of predicting MCS separation shock, ${ }^{2,3)}$ but these methods give an empirical prediction based on a statistical analysis of previous experiments and may not agree with experimental results. This paper presents an analytical method developed to estimate the magnitude of separation shock from MCS. The model is based on a mathematical algorithm and numerical simulation, not on prior statistical or empirical data, and is verified by newly collected data from various configurations of actual systems.

First, data were collected from numerous experiments by the use of several MCS engineering models for actual flight hardware. Next, the experimental results were analyzed, and the likely mechanism by which the separation shock is generated was determined. A predictive model was proposed from these results.

\section{Experimental Results}

\subsection{Description of MCS}

A diagram of MCS is shown in Fig. 1. Generally, MCS consists of two cylinders and a belt clamp. Each cylinder has an outside flange and usually an inside ring at the junction plane to prevent the cylinder from buckling.

The belt has a V-shaped slot on the inside to fasten the flanges of the cylinders together. The belt is usually called the "Marman band" or "Marman clamp" or "V clamp," and the name MCS derives from this band.

To form the joint, the flanges of the upper and lower cylinders are placed together and inserted into the slot of the Marman band. The Marman band is then stretched with one or more tensioners, typically bolt and nut, and the joint is fastened. To separate the joint, the Marman band is cut by the use of pyrotechnics, typically a device actuated by gunpowder. Generally, redundant pyrotechnics are used with two or more cut points on the Marman band. In Fig. 1, two cut points are used.

MCS usually has springs to accelerate separation and to prevent the lower cylinder from colliding with the upper cylinder, since a residual thrust of a solid rocket booster of

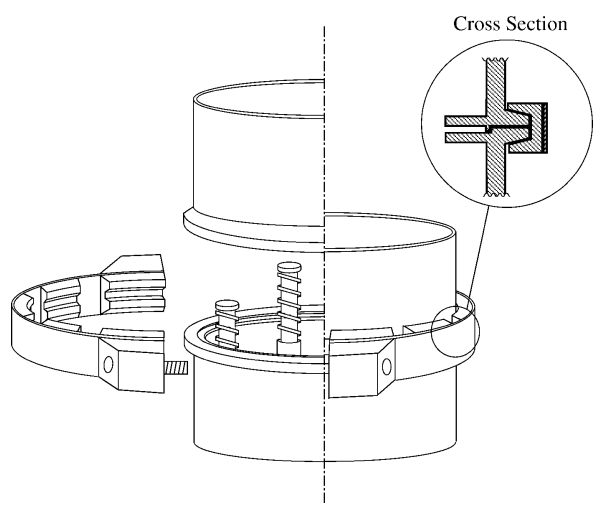

Fig. 1. Diagram of the Marman Clamp System.

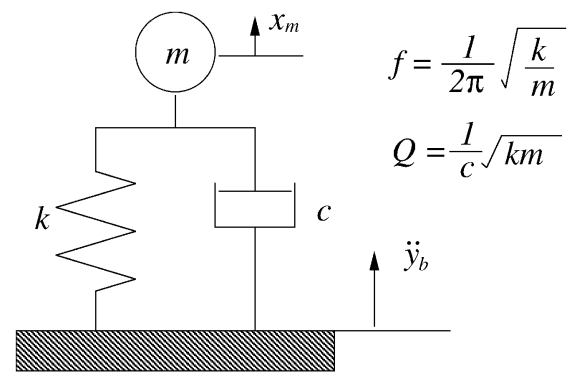

$\mathrm{SRS}=\max \left(\left|\ddot{x}_{m}\right|\right)$

Fig. 2. One degree of freedom system for calculating SRS.

the lower stage might cause a collision of stages.

\subsection{Shock response spectrum}

Separation shock typically refers to the acceleration generated when separation occurs. Satellite instrumentation is designed to accommodate that acceleration. In this paper, separation shock (or simply shock) is used to mean the acceleration.

Since it is difficult to pick up required information for design from the acceleration with a waveform in the time domain, the Shock Response Spectrum index (SRS) is generally used to evaluate the acceleration. ${ }^{2,3)}$ To compute SRS, we consider a system with one degree of freedom shown in Fig. 2. SRS is the maximum response acceleration $\left|\ddot{x}_{\mathrm{m}}\right|$ generated in a mass, $m$, as a function of the natural frequency of the system $f=\sqrt{k / m} / 2 \pi$. When an acceleration, $\ddot{y}_{\mathrm{b}}$, is input to the system, the value of SRS at a frequency $f, S(f)$, can be derived from following formulas.

$$
\begin{gathered}
m\left(\ddot{x}_{\mathrm{m}}-\ddot{y}_{\mathrm{b}}\right)+c\left(\dot{x}_{\mathrm{m}}-\dot{y}_{\mathrm{b}}\right)+k\left(x_{\mathrm{m}}-y_{\mathrm{b}}\right)=-m \ddot{y}_{\mathrm{b}} \\
S(f)=\operatorname{Max}\left(\left|\ddot{x}_{\mathrm{m}}\right|\right)
\end{gathered}
$$

For the system's resonance magnification, $Q=\sqrt{\mathrm{km}} / \mathrm{c}$, $Q=10$, and a natural frequency range $100(\mathrm{~Hz}) \leq f \leq$ $10,000(\mathrm{~Hz})$ are often used. ${ }^{2,3)}$ We employ these same values in this paper.

Our experiments were conducted by causing a separation of MCS in situations identical to actual launch conditions and by measuring the resultant acceleration. To obtain data that characterized separation shock, the experiments were carried out under a variety of conditions. The items described in the following sections were examined in detail.

\subsection{The relationship between separation shock and the distance from the pyrotechnics}

The pyrotechnics use gunpowder to sever the Marman band and generate a large shock when activated. This shock is called "pyrotechnic shock." It is very large value near of the pyrotechnics (within millimeters), and immeasurable with common accelerometers (about $10^{4}(\mathrm{G})$ shock proof models).

Since MCS is a thin cylindrical shell, the pyrotechnic shock spreads two-dimensionally along the cylindrical surface. This could imply that the strength of the pyrotech- 


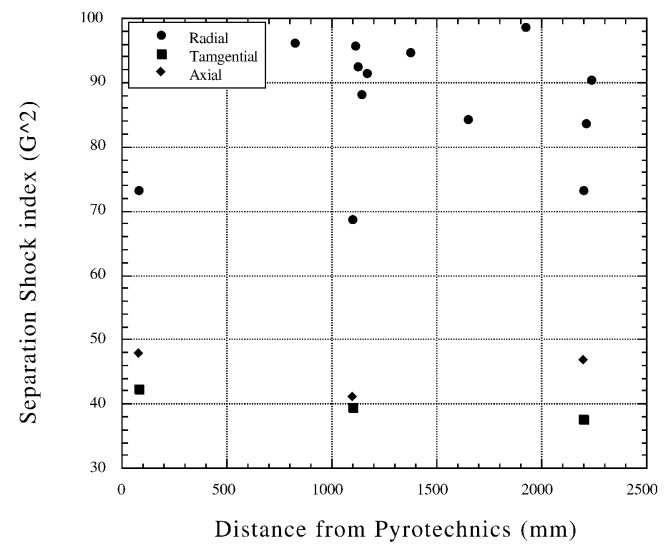

Fig. 3. Plot of the separation shock index against distance from pyrotechnics.

nic shock at a site is inversely proportional to the distance from the pyrotechnics. To determine if pyrotechnic shock is the dominant component of separation shock from MCS, we plotted in Fig. 3 an index of separation shock against distance from the pyrotechnics. In that figure, the separation shock index is not shown as SRS, but as the squared time integral of shock acceleration. The data are segmented by the direction of the measured acceleration (cylindrical radial, tangential, and axial). Figure 3 shows that the separation shock is not in inverse proportion to the distance from pyrotechnics, but it is nearly constant. This reveals that at the point where the satellite instrumentation is mounted $(50 \mathrm{~mm}$ or more from the pyrotechnics), pyrotechnic shock is not the dominant separation shock component when MCS is being used.

\subsection{The relationship between separation shock and Marman band tension}

In MCS, a Marman band under considerable tension is used to connect the stages. To determine the effect of this tension on the separation shock using MCS, four experiments were carried out with various band tensions. Two experiments were conducted with a high-tension band and two with a low-tension band. The ratio of low to high tensions averaged approximately 0.57 . Each result was converted to SRS and averaged for each of two experiments at different tensions. Next, the ratio of the mean results for each frequency was calculated. That is, if each value of SRS at a frequency $f$ equals $S_{1}(f), S_{2}(f)$ (the results of experiments with high-tension values) and $S_{3}(f), S_{4}(f)$ (the results of experiments with low-tension values), the ratio of SRS $R(f)$ at $f$ is derived from

$$
R(f)=\frac{S_{3}(f)+S_{4}(f)}{S_{1}(f)+S_{2}(f)}
$$

The values calculated for the ratio $R(f)$ are shown in Fig. 4 for each direction (radial, tangential, and axial).

Figure 4 shows that the ratio of SRS (especially in the radial and axial directions) is near 0.57 , therefore the radial and axial separation shock of MCS is considered to be proportional to the Marman Band tension. It may be further con-

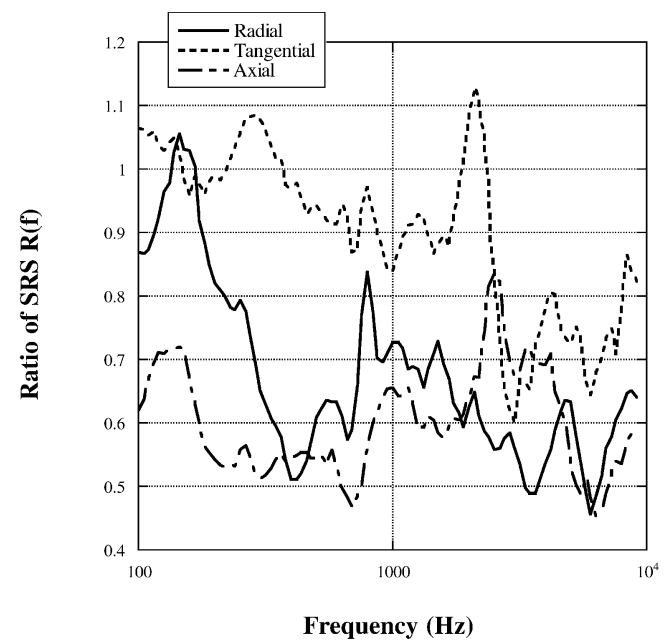

Fig. 4. Plots of SRS ratio caused by a decrease in the Marman band tension.

cluded that the vibration generated by the release of strain energy dominates in the radial and axial separation shock of MCS. On the other hand, when separation shock in the tangential direction is considered, Marman band tension had no effect on the ratio of SRS in the low-frequency region and only a slight effect in the high-frequency region. Thus vibration generated by the release of strain energy may not be dominant in the tangential separation shock of MCS.

2.5. The relation between separation shock and the mass of the Marman band

To determine the effect of the mass of Marman band on separation shock, two experiments were conducted in which the mass of the Marman band was increased. The total mass used in the experiments was approximately twice that of a normal Marman band. The ratio of SRS calculated by the use of methods outlined in the previous section is shown in Fig. 5.

Figure 5 reveals that the ratio of SRS for all directions is less than 1.0 across almost all frequency regions. It turns out

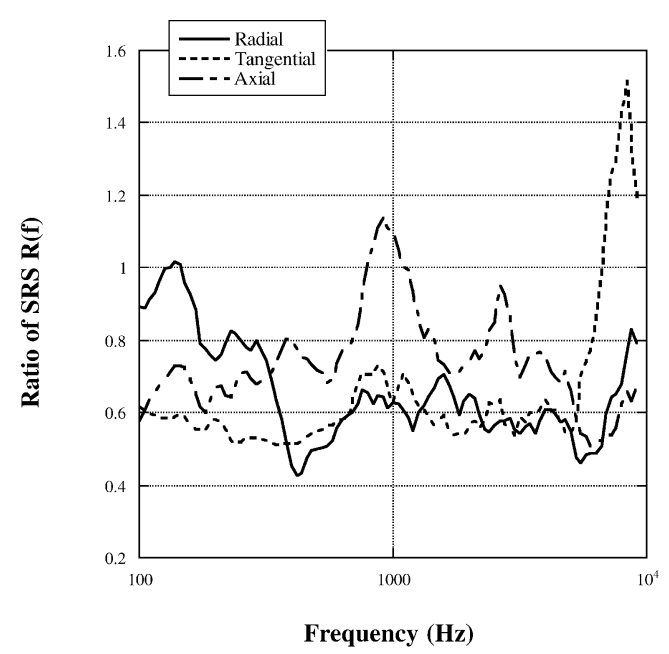

Fig. 5. Plots of SRS ratio caused by an increase of the Marman band mass. 


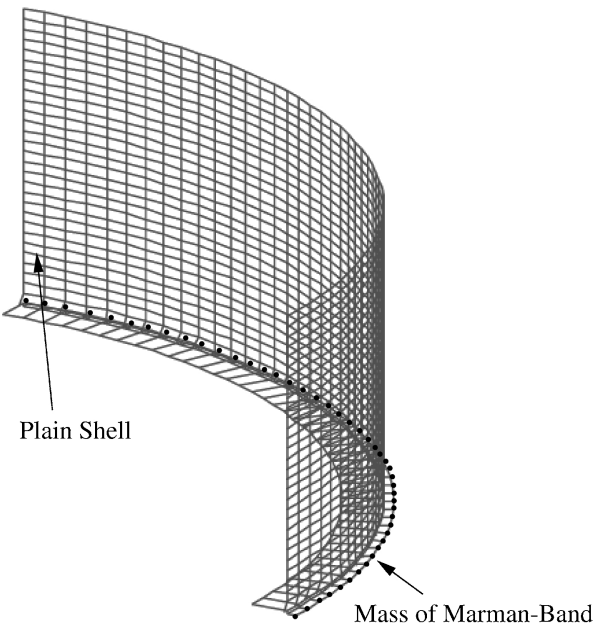

Fig. 6. Finite element model of MCS.

that the separation shock of MCS is decreased by an increase in the mass of the Marman band.

\section{Analysis Method Using FEM}

\subsection{Modeling of MCS}

The experimental results described in the previous section 2 show that the structural vibration generated by the release of strain energy might be the dominant component of separation shock of MCS, especially in the radial and axial directions. To analyze the vibration, we modeled MCS by using the Finite Element Method (FEM). MCS was modeled as shown in Fig. 6 by the use of plain shell elements, and in consideration of the symmetry of this problem, we modeled only a quarter of MCS.

\subsection{Modeling of the Marman band}

The treatment of the Marman band is problematic. Figure 5 demonstrates that its mass has an effect on the separation shock of MCS, so it is necessary to model the effect of this mass to create a predictive model. If the Marman band is modeled by the use of only FEM, this problem will become the contact problem in which two or more structures contact mutually and exert force on each other. Many solutions to the contact problem have been proposed, ${ }^{4,5)}$ but the contact problem is statically indeterminate in essence, and iteration is needed to provide any solution. In the solving of a dynamic problem, in this case a vibration calculation, the number of calculations would become impracticably large. In this paper we hypothesized that the stiffness of the Marman band is negligible, which avoids the contact problem. When this hypothesis is used, the role of the Marman band in the separation of MCS is simply modeled as follows in sequence.

1. Force to fasten MCS because of the Marman band tension until the Marman band is severed $(t<0)$

2. Mass generating the inertial force until time $\tau(0 \leq$ $t<\tau$ )

3. No effect on $\operatorname{MCS}(t \geq \tau)$

\subsection{Force resulting from the Marman band tension}

The Marman band tension is almost constant and the distribution of the initial force resulting from the tension is almost axisymmetric. However, we treated the Marman band tension not as a constant, but as a variable approximate to an actual distribution in consideration of the frictional force between the flanges of MCS and the Marman band.

By taking a $\theta$ coordinate along the circumference of the cylinder, the position of the Marman band tensioner is set at $\theta=0$ (see Fig. 7). The tension of the Marman band at $\theta$ is set at $T(\theta)$, the radius of Marman band is set at $a$, the radial direction force on the Marman band per unit length along the circumference is set at $p(\theta)$, and the coefficient of friction for the force that applies in the tangential direction between the Marman band and flanges is set at $\mu$. The following formulas are derived from the equilibrium of forces in the radial and tangential directions that affect a small element of the Marman band.

$$
\left[T\left(\theta+\frac{d \theta}{2}\right)+T\left(\theta-\frac{d \theta}{2}\right)\right] \sin \left(\frac{d \theta}{2}\right)=p(\theta) a d \theta
$$

$$
\left[T\left(\theta+\frac{d \theta}{2}\right)-T\left(\theta-\frac{d \theta}{2}\right)\right] \cos \left(\frac{d \theta}{2}\right)=-\mu p(\theta) a d \theta
$$

Where $d \theta \cong 0$, the following formulas are derived from Eqs. (4) and (5).

$$
\begin{gathered}
\frac{d T(\theta)}{d \theta}=-\mu T(\theta) \\
p(\theta)=\frac{T(\theta)}{a}
\end{gathered}
$$

From Eqs. (6) and (7) the tension distribution (which decreases exponentially) is obtained.

$$
T(\theta)=T_{0} \exp (-\mu \theta)
$$

Here the initial force resulting from the Marman band tension consists of the force $p(\theta)$ in the radial direction and the force $\mu p(\theta)$ in the tangential direction derived from Eqs. (7) and (8).

In Eq. (8), $T_{0}$ and $\mu$ are unknown. To determine these values, we fit Eq. (8) to an actual distribution measured by experiment, using the least squares method. We show an

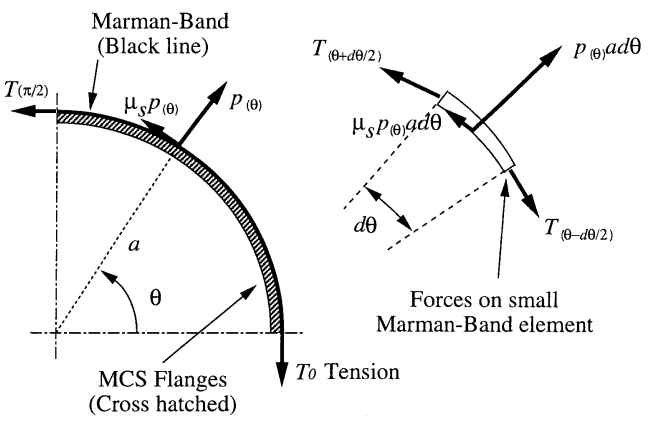

Fig. 7. Marman band tension model. 


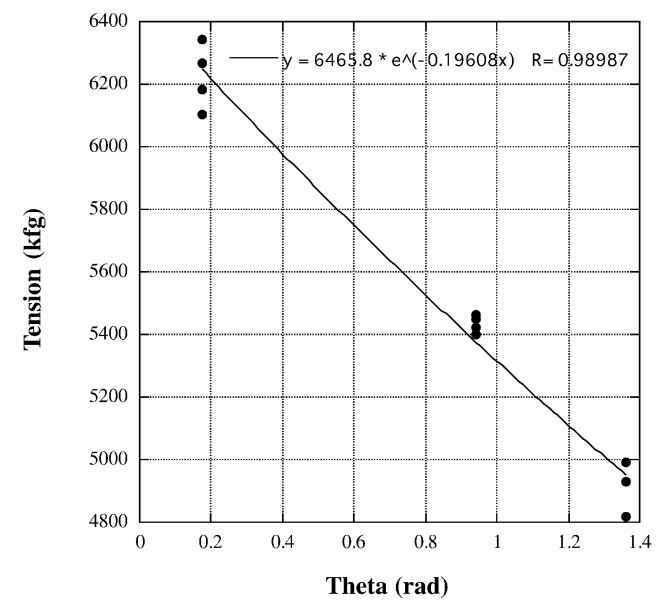

Fig. 8. Comparison of exponentially decreasing and actual tension distribution.

example of the fitting result in Fig. 8. In Fig. 8, $T_{0}$ equals 6,470 (kgf) and $\mu$ equals 0.196 .

\subsection{Modeling of the Marman band mass}

Since Fig. 5 shows that the mass of the Marman band has an effect on the separation shock of MCS, the mass must be taken in the model. It is modeled by the use of a mass element distributed on the end of the FEM model (See Fig. 6). To simplify the computation, each mass is fixed on the same node where it was fixed in the initial state from $t=0$ to $t=\tau$. Here $\tau$ is the time when the Marman band detaches from the MCS.

We approximate $\tau$ as a quarter of a minimum-order axisymmetric natural vibration cycle of MCS in this paper. The cycle can be computed from Eq. (9) in the next section by the use of the eigenvalue analysis. This is because the minimumorder axisymmetric natural vibration mode is the most displaced mode in the initial state, since the distribution of the initial force is almost axisymmetric. And also because a mass put on a spring detaches from the spring at a quarter of the natural vibration cycle of the 1 DOF system consisting of the mass and the spring when the shortened spring is released from the stationary state at $t=0$.

\subsection{Formulation}

The equations of motion based on FEM are as follows.

$$
\begin{array}{ll}
\left(M+M_{\mathrm{b}}\right) \ddot{\boldsymbol{x}}+K \boldsymbol{x}=0 & (t<\tau) \\
M \ddot{\boldsymbol{x}}+K \boldsymbol{x}=0 & (t \geq \tau)
\end{array}
$$

Here $M$ is a mass matrix of MCS without the Marman band, $M_{\mathrm{b}}$ is a mass matrix of the Marman band itself, $K$ is a stiffness matrix of MCS, and $\boldsymbol{x}$ is a nodal displacement vector. The initial state of $\boldsymbol{x}$ at $t=0$ is given as follows.

$$
\begin{gathered}
K \boldsymbol{x}=\boldsymbol{F}_{0} \\
\dot{\boldsymbol{x}}=0
\end{gathered}
$$

Here $\boldsymbol{F}_{0}$ is the initial force vector whose distribution is derived by discretizing $p(\theta)$ and $\mu p(\theta)$ from Eqs. (7) and (8) with FEM. $\boldsymbol{x}$ and $\dot{\boldsymbol{x}}$ are continuous at $t=\tau$.
These equations were solved by the use of the modal analysis method. Modal damping was applied and uniformly set at $0.5 \%$.

\subsection{Computational results}

Comparisons between experimental results and computational results based on our hypotheses are shown in Figs. 9 (radial), 10 (tangential), and 11 (axial), and in Figs. 12 (radial), 13 (tangential), and 14 (axial). The experimental results were typical sample data from the experiments conducted with an engineering model of MCS for the actual flight hardware of a certain satellite. The distribution of Marman band tension in this experiment is shown in Fig. 8 as an example. The results in Figs. 9, 10, and 11 are waveforms of acceleration and computational results time shifted 0.0037 (s) to adjust to the experimental result. In Figs. 12, 13 , and 14 , the results are transformed into SRS.

Figures 9, 11, 12, and 14 show that the separation shock can be estimated with sufficient accuracy in the radial and axial directions with our hypotheses, both as a waveform and as an SRS. For tangential shock, however, Figs. 10 and 13 show that the computational results are smaller than the experimental results. This is very likely that a factor other than structural vibration, which is generated by the release of strain energy, dominates the tangential separation shock, as illustrated in Fig. 4.

Computational results in which the mass of Marman band is ignored are also shown in Figs. 12, 13, and 14. The results are computed as $M_{\mathrm{b}}=0$ and plotted "without mass" in the figures. In Figs. 12 and 14, the results without mass show higher shock than observed in the experimental results. The results that include the effect of the mass of the Marman band agree well with the experimental results. This shows that the simple model can simulate the effect of the Marman band mass during MCS separation.

Results using statistical predictions in Refs. 2 and 3 are also shown in Figs. 12, 13, and 14. However, since this method gives only one result not segmented by the direction of separation shock, the same results are shown in the figures. Figures 12, 13, and 14 show that in this case the difference between experimental results and empirical prediction is larger than that of experimental results and our computational results.

We conducted many other experiments using this MCS of a certain satellite, and also using other two MCSs, engineering models for actual flight hardware, and another MCS, test hardware made for experiment. These MCSs cover a wide geometrical range, for example, the radius of the smallest MCS is about $6.5(\mathrm{~cm})$ and that of the largest one is about $70(\mathrm{~cm})$. The results are not shown as any figures in this paper because of the lack of space. In a summary of these results, however, each computational result agreed well with the experimental results in the radial and axial directions and showed smaller shock in the tangential direction. 

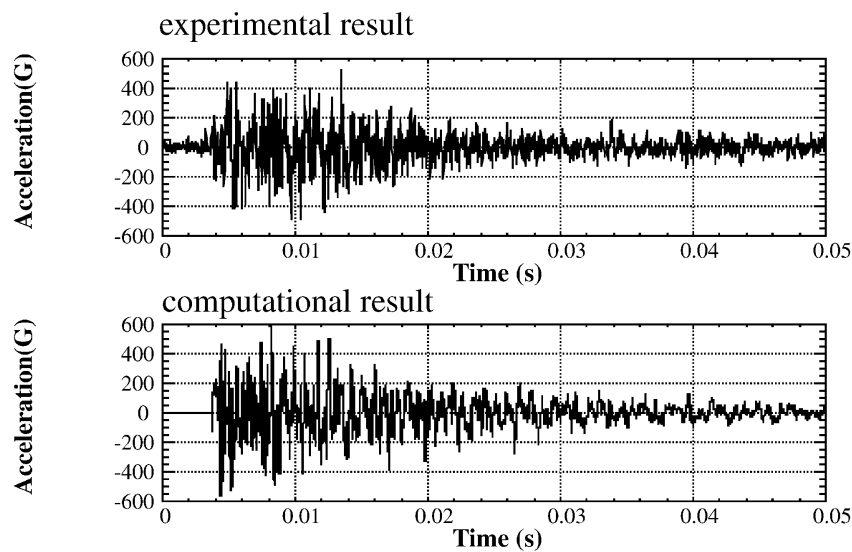

Fig. 9. Computational results of radial separation shock with FEM (waveform).
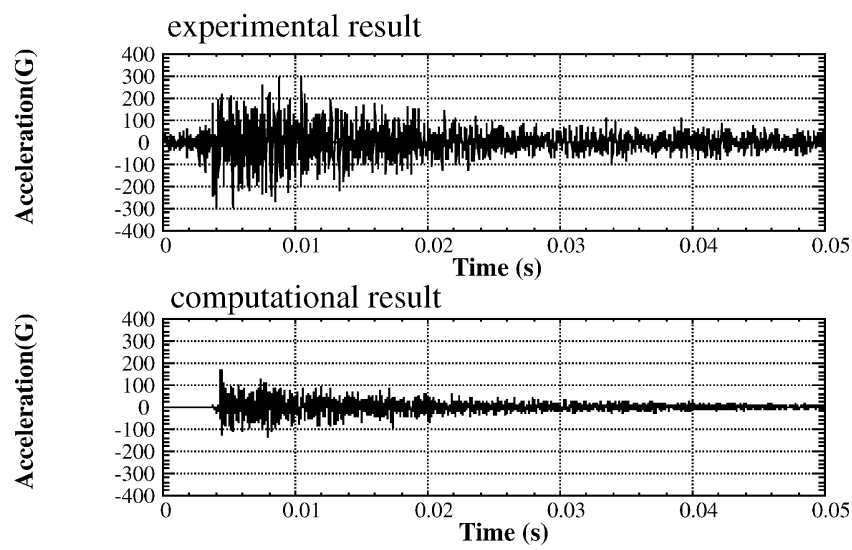

Fig. 10. Computational results of tangential separation shock with FEM (waveform).
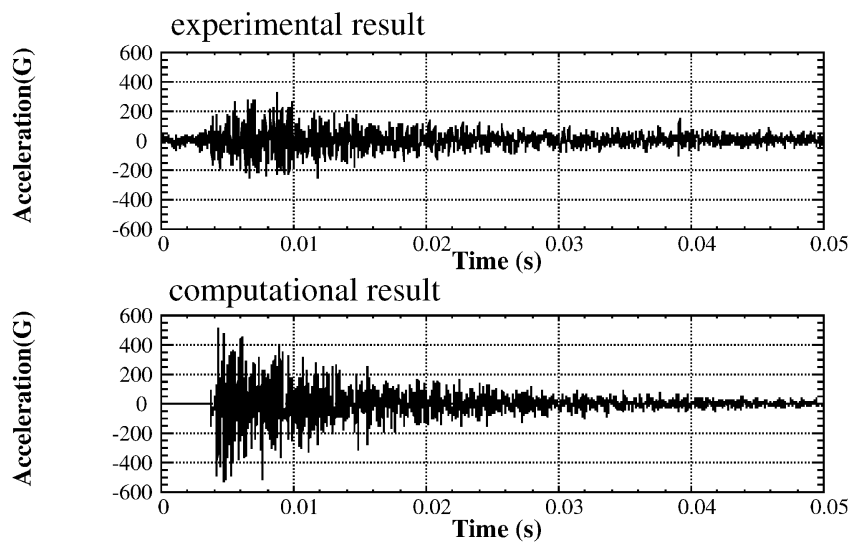

Fig. 11. Computational results of axial separation shock with FEM (waveform).

\section{Analysis Method Using the Equation of an Axisym- metric Shell}

We collected the three experimental results and the three computational results in Figs. 12, 13, and 14 and show them

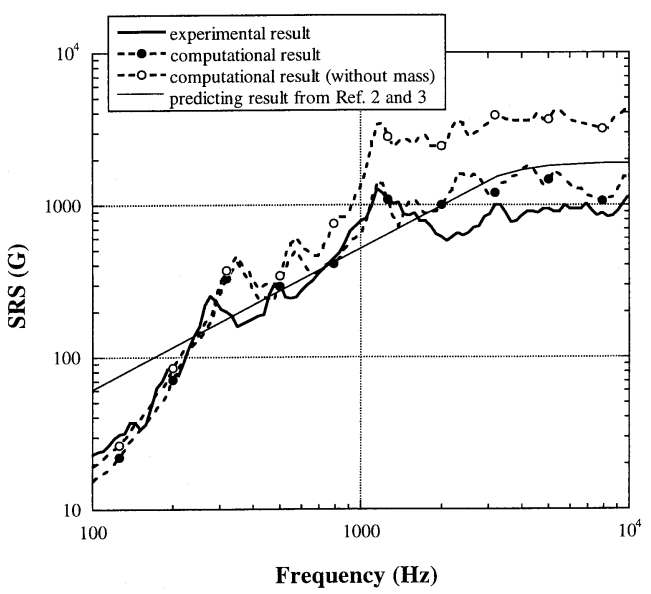

Fig. 12. Computational results of radial separation shock with FEM (SRS).

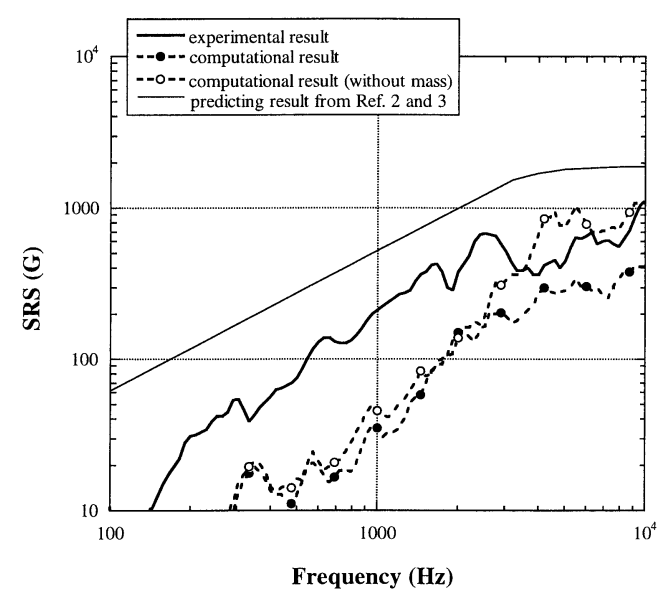

Fig. 13. Computational results of tangential separation shock with FEM (SRS).

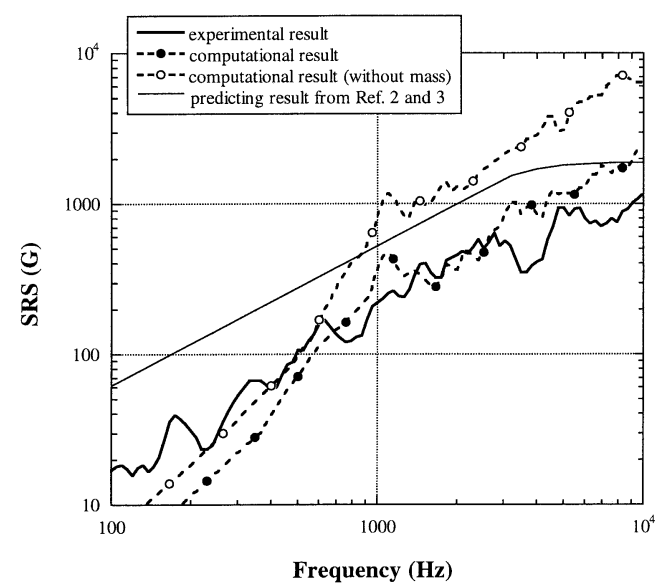

Fig. 14. Computational results of axial separation shock with FEM (SRS).

in Fig. 15. In Fig. 15, a peak near 1,135 (Hz) in the radial direction seemed to be most significant because it showed the largest value among the three experimental results. An analysis of the computational results shows that axisymmet- 


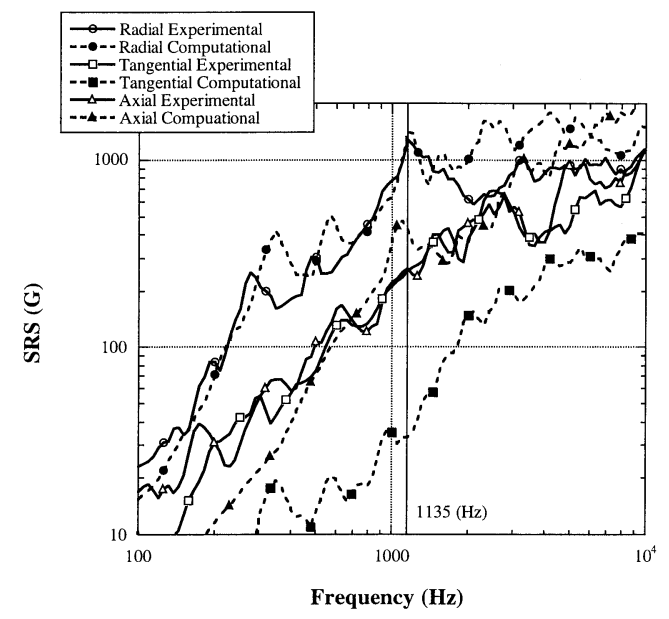

Fig. 15. Comparison between experimental and computational results with FEM (SRS).

ric modes caused this peak. Therefore we propose another method in which only the axisymmetric modes are used to estimate this peak, which simplifies the calculation. Furthermore, we try to construct the method by using the equation of an axisymmetric shell, because solutions with literal notation (not numerical) can be derived from these methods, and the solutions will help us to understand the effects of design parameters (thickness, radius, modulus for example) on the separation shock in a future analysis.

\subsection{Formulation}

Each cylinder of MCS is essentially a cylindrical shell with an outside flange and occasionally an inside ring. In the hypothesis that these attachments and the mass of the Marman band converge at the end of the cylinder and that their distribution is axisymmetric, the mass of the outside flange and inside ring per unit length along the circumference is set at $\rho_{\mathrm{c}}$. Similarly, the spring constant relative to the radial deformation of the outside flange and inside ring per unit length along the circumference is set at $k_{\mathrm{c}}$, the mass of the Marman band per unit length along the circumference is set at $\rho_{\mathrm{b}}$, and the length of the cylindrical shell is set at l. Each cylinder of MCS can be considered a homogeneous cylindrical shell with thickness $h$ and radius $a$. Its material density is set at $\rho$, with Young's modulus $E$ and Poisson's ratio $v$. With an $x$ coordinate in the axial direction and a $z$ coordinate in the radial direction, the end of the cylindrical shell at which $\rho_{\mathrm{c}}, k_{\mathrm{c}}$, and $\rho_{\mathrm{b}}$ converge is set at $x=l$, and the other end is set at $x=0$ (See Fig. 16).

Here $w$, the displacement in the $z$ direction of the middle surface of the shell, satisfies the following equation on the assumption that we can use the Donnell's equations ${ }^{6}$ because the shell is axisymmetric and that the motion of the shell is a free vibration.

$$
\frac{\partial^{2}}{\partial x^{2}}\left(D \frac{\partial^{2} w}{\partial x^{2}}\right)+\frac{E h}{1-v^{2}}\left(1-v^{2}\right) \frac{w}{a^{2}}=-\mu_{\mathrm{s}} \frac{\partial^{2} w}{\partial t^{2}}
$$

Here $D$ is the bending stiffness of the shell, and $\mu_{\mathrm{s}}$ is the mass of the shell per unit area. $D$ and $\mu_{\mathrm{s}}$ are defined as

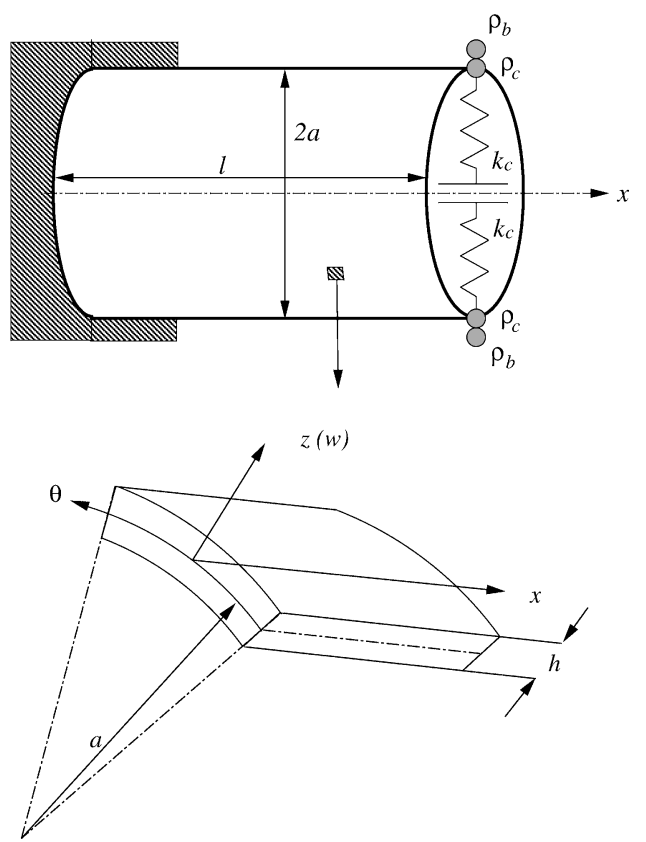

Fig. 16. Coordinates on the cylindrical shell.

follows.

$$
\begin{gathered}
D \equiv \frac{E h^{3}}{12\left(1-v^{2}\right)} \\
\mu_{\mathrm{s}} \equiv \rho h
\end{gathered}
$$

Next we consider boundary conditions. The boundary at $x=0$ is fixed as in the experiments. At the other boundary, at $x=l, \rho_{\mathrm{c}}, k_{\mathrm{c}}$, and $\rho_{\mathrm{b}}$ converge, where $\rho_{\mathrm{b}}$ is the mass of the Marman band. Assuming that $\rho_{\mathrm{b}}$ has the same role as the mass of the Marman band described in section 3.2., the boundary conditions of the shell are derived as follows.

$$
\begin{gathered}
w=\frac{\partial w}{\partial x}=0 \quad \text { at } \quad x=0 \\
\frac{\partial}{\partial x}\left(D \frac{\partial^{2} w}{\partial x^{2}}\right)=\left\{\begin{array}{lll}
k_{\mathrm{c}} w+\left(\rho_{\mathrm{b}}+\rho_{\mathrm{c}}\right) \frac{\partial^{2} w}{\partial t^{2}} & \text { at } \quad x=l, t<\tau \\
k_{\mathrm{c}} w+\rho_{\mathrm{c}} \frac{\partial^{2} w}{\partial t^{2}} & \text { at } & x=l, t \geq \tau
\end{array}\right. \\
D \frac{\partial^{2} w}{\partial x^{2}}=0 \quad \text { at } \quad x=l
\end{gathered}
$$

\subsection{Computational results}

Equation (13) was solved with the modal analysis method. Modal damping was applied and uniformly set at $0.5 \%$. Computational results are shown in Figs. 17 (waveform) and 18 (SRS). In these figures, the experimental and computational results with FEM are also shown for comparison. With the addition of a supplementary explanation, this method can estimate only the separation shock in the radial direction.

In Fig. 17, the computational result with the equation of an axisymmetric shell shows a sparser waveform than the experimental result and the computational result using FEM. This is because the computational result using the equa- 

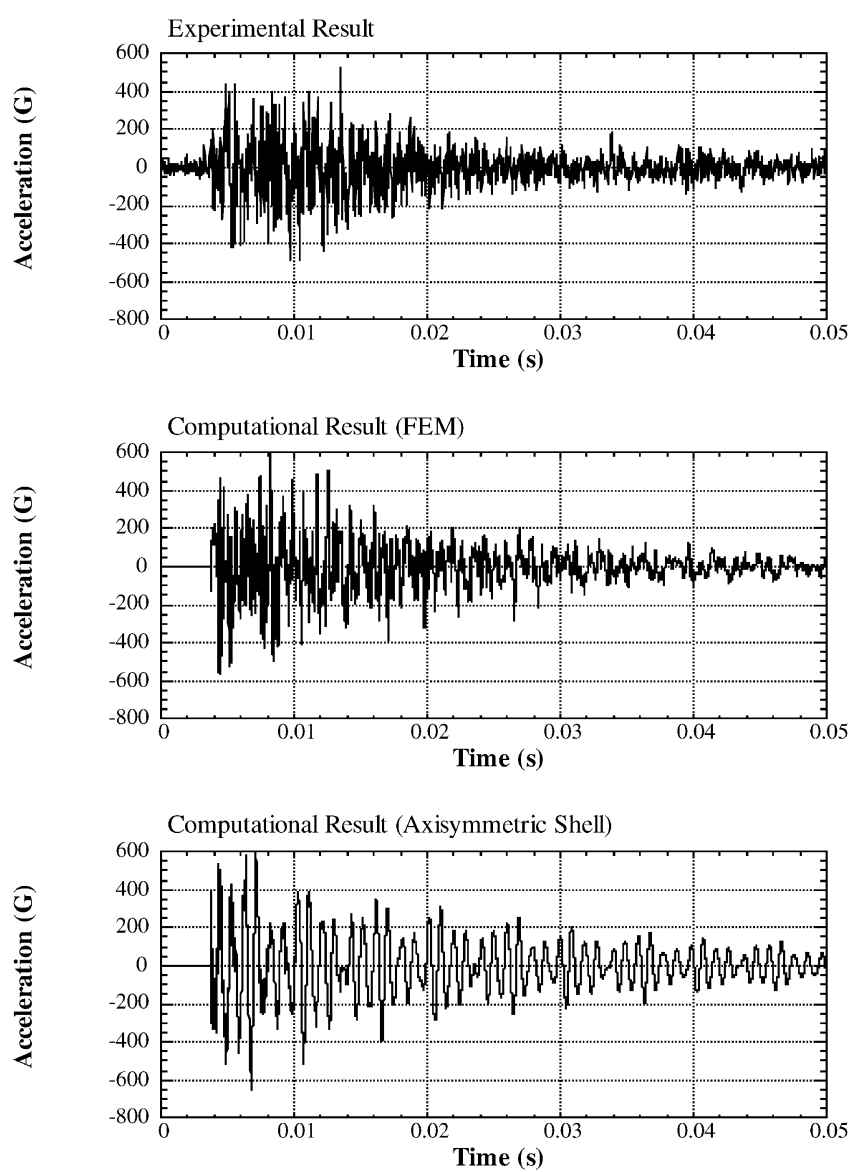

Fig. 17. Computational results of radial separation shock with the equation of an axisymmetric shell (waveform).

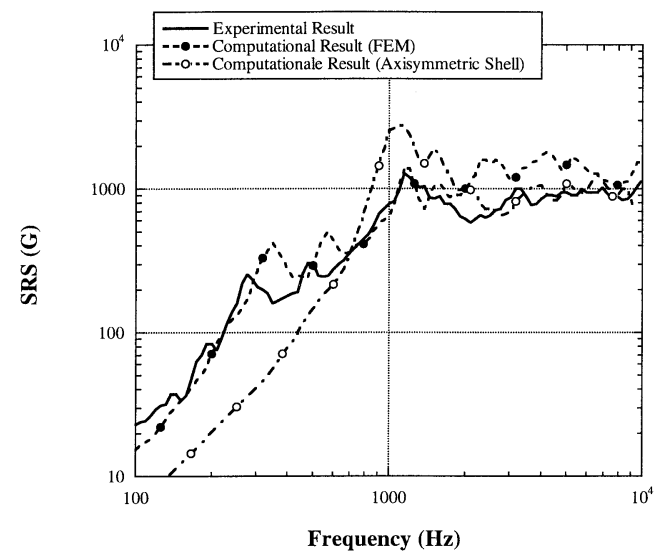

Fig. 18. Computational results of radial separation shock with the equation of an axisymmetric shell (SRS).

tion of an axisymmetric shell includes fewer modes than the experimental results and FEM computational results. In Fig. 18, however, by using the equation of an axisymmetric shell we can estimate an approximate magnitude and frequency of the peak that shows the largest value at 1,135 (Hz) in the experimental result. Especially by appending an example of a solution with literal notation, the frequency of the peak almost equals the first natural frequency of the shell $f_{\mathrm{p}}=1,174(\mathrm{~Hz})$ as follows.

$$
f_{\mathrm{p}}=\frac{1}{2 \pi} \sqrt{\frac{D}{\mu_{\mathrm{s}}}} \sqrt{\frac{\lambda^{4}}{l^{4}}+\frac{12\left(1-v^{2}\right)}{a^{2} h^{2}}}
$$

Here $\lambda$ is the minimum positive solution of the following equation.

$$
\begin{aligned}
& -2\left(\frac{\lambda}{l}\right)^{3}\left\{\left(\frac{\lambda}{l}\right)^{3}(1+\cosh \lambda \cos \lambda)\right. \\
& +\left(\frac{k_{\mathrm{c}}}{D}-\frac{\rho_{\mathrm{c}}}{\mu_{\mathrm{s}}} \frac{12\left(1-v^{2}\right)}{a^{2} h^{2}}\right)(\cosh \lambda \sin \lambda-\cos \lambda \sinh \lambda) \\
& \left.-\frac{\rho_{\mathrm{c}}}{\mu_{\mathrm{s}}}(\sin \lambda \cosh \lambda-\cos \lambda \sinh \lambda)\left(\frac{\lambda}{l}\right)^{4}\right\}=0
\end{aligned}
$$

\section{Conclusion}

The experimental results demonstrate that the dominant radial and axial components of MCS separation shock are structural vibration generated by the release of strain energy. Based on the experimental results, a simplified model of the Marman band effects on separation shock of MCS and a predictive method that uses a Finite Element Method (FEM) are proposed. The results of estimates are largely in agreement with the experimental results for measured radial and axial separation shock. Furthermore, an estimation method based on the equation of an axisymmetric shell is presented. A solution with literal notation is derived from the method, and the results of the estimation are mostly in agreement with the experimental results at the largest SRS value of the separation shock of MCS. Future investigations should focus on the dominant component of tangential separation shock and propose an accurate model for its estimation.

\section{References}

1) Marman Clamp System Design Guidelines, Guideline No. GD-ED2214, NASA Goddard Space Flight Center, 2000.

2) Kacena, W., McGrath, M. and Rader, A.: Aerospace System Pyrotechnic Shock Data, Vol. I-Vol. VII, NASA CR 116419, Goddard Space Flight Center, 1970.

3) Pyroshock Test Criteria, NASA Technical Standard, NASA-STD7003, May 18, 1999.

4) Chandrasekaran, N., Haisler, W. E. and Goforth, R. E.: A Finite Element Solution Method for Contact Problems with Friction, Int. J. Numer Meth. Eng., 24 (1987), pp. 477-484.

5) Gallego, F. J. and Anza, J. J.: A Mixed Finite Element Model for the Elastic Contact Problem, Int. J. Num. Meth. Eng., 28 (1989), pp. 12491264.

6) Donnell, L. H.: Stability of Thin-Walled Tubes under Torsion, NACA TR 479, 1934. 